\title{
Polymer desorption under pulling: a novel dichotomic phase transition
}

\author{
S. Bhattacharya ${ }^{1}$, V. G. Rostiashvili ${ }^{1}$, A. Milchev ${ }^{1,2}$, and T.A. Vilgis ${ }^{1}$ \\ 1 Max Planck Institute for Polymer Research, \\ 10 Ackermannweg, 55128 Mainz, Germany \\ ${ }^{2}$ Institute for Physical Chemistry, Bulgarian Academy of Sciences, 1113 Sofia, Bulgaria
}

\begin{abstract}
We show that the structural properties and phase behavior of a self-avoiding polymer chain on adhesive substrate, subject to pulling at the chain end, can be obtained by means of a Grand Canonical Ensemble (GCE) approach. We derive analytical expressions for the mean length of the basic structural units of adsorbed polymer, such as loops and tails, in terms of the adhesive potential and detachment force, and determine values of the universal exponents which govern their probability distributions. Most notably, the hitherto controversial value of the critical adsorption exponent $\phi$ is found to depend essentially on the interaction between different loops. The chain detachment transition turns out to be of the first order, albeit dichotomic, i.e., no coexistence of different phase states exists. These novel theoretical predictions and the suggested phase diagram of the adsorption-desorption transformation under external pulling force are verified by means of extensive Monte Carlo simulations.

PACS numbers: 05.50.+q, 68.43.Mn, 64.60.Ak, 82.35.Gh, 62.25.+g
\end{abstract}


Introduction - The manipulation of single polymer chains has turned recently into an important method for understanding their mechanical properties and characterization of the intermolecular interactions [1, 2]. Such manipulation is mainly triggered by the progress in atomic force microscopy (AFM) [3] as well as by the development of optical/magnetic tweezers technique [4]. This rapid development has been followed by theoretical considerations, based on the mean - field approximation [5], which provide important insight into the mechanism of polymer detachment from adhesive surfaces under the external force pulling. A comprehensive study by Skvortsov et al. [6] examines the case of a Gaussian polymer chain. We also note here the close analogy between the force detachment of adsorbed chain and the unzipping of a double - stranded DNA. Recently, DNA denaturation and unzipping have been treated by Kafri et al. [7] using the Grand Canonical Ensemble (GCE) approach [8] as well as Duplantier's analysis of polymer networks of arbitrary topology [9]. An important result for the properties of adsorbed macromolecule under pulling turns to be the observation [7] that the universal exponents (which govern polymer statistics) undergo renormalization due to excluded volume effects, leading thus to a change of the order of DNA melting transition from second to first order. In this Letter we use similar methods to describe the structure and detachment of a single chain from a sticky substrate when the chain end is pulled by external force.

Single chain adsorption - Starting with the conventional (i.e., force-free) adsorption, we recall that an adsorbed chain is build up from loops, trains, and a free tail. One can treat statistically these basic structural units by means of the GCE approach where the lengths of the buildings blocks are not fixed but may rather fluctuate. The GCE-partition function is then given by

$$
\Xi(z)=\sum_{N=0}^{\infty} \Xi_{N} z^{N}=\frac{V_{0}(z) Q(z)}{1-V(z) U(z)},
$$

where $z$ is the fugacity and $U(z), V(z)$, and $Q(z)$ denote the GCE partition functions of loops, trains and tails, respectively. The building block adjacent to the tethered chain end is allowed for by $V_{0}(z)=1+V(z)$. The partition function of the loops is defined as $U(z)=\sum_{n=1}^{\infty}\left(\mu_{3} z\right)^{n} / n^{\alpha}$, where $\mu_{3}$ is the $3 d$ connective constant and $\alpha$ is the exponent which governs surface loops statistics. It is well known that for an isolated loop $\alpha=$ $1-\gamma_{11} \approx 1.39[10]$. We will argue below that $\alpha$ changes value due to the excluded volume interaction between a loop and the rest of the chain. The train GCE-partition function 
reads $V(z)=\sum_{n=1}^{\infty}\left(\mu_{3} w z\right)^{n} / n^{1-\gamma_{d=2}}$ whereby one assumes that each adsorbed segment gains an additional statistical weight $w=\exp (\epsilon)$ with the dimensionless adsorption energy $\epsilon=\varepsilon / k_{B} T$. Eventually, the GCE partition function for the chain tail is defined by $Q(z)=$ $1+\sum_{n=1}^{\infty}\left(\mu_{3} z\right)^{n} / n^{\beta}$. For an isolated tail $\beta=1-\gamma_{1} \approx 0.32$ [10] but again the excluded volume interactions of a tail with the rest of the chain increase the value of $\beta$. Using the generating function method [11], $\Xi_{N}$ is obtained as $\Xi_{N}=\left(z^{*}\right)^{-N}$ where the pole $z^{*}$ is given by the condition $V\left(z^{*}\right) U\left(z^{*}\right)=1$ so that the free energy is $F=k_{B} T N \ln z^{*}$ and the fraction of adsorbed monomers $n=-\partial \ln z^{*} / \partial \ln w$. In terms of the so called polylog function, which is defined as $\Phi(\alpha, z)=\sum_{n=1}^{\infty} z^{n} / n^{\alpha}$ [12], the equation for $z^{*}$ reads

$$
\Phi\left(\alpha, \mu_{3} z^{*}\right) \Phi\left(\lambda, \mu_{2} w z^{*}\right)=1 \text {. }
$$

A nontrivial solution for $z^{*}$ in terms of $w$ (or the adsorption energy $\epsilon$ ) appears at the critical adsorption point $(\mathrm{CAP}) w=w_{c}$ where $w_{c}$ is determined from $\left.\zeta(\alpha) \Phi\left(1-\gamma_{d=2}, \mu_{2} w_{c} / \mu_{3}\right)\right)=1$ as $z^{*}=1 / \mu_{3}$ and $\zeta(\alpha)$ is the Riemann function. In the vicinity of the CAP the solution attains the form

$$
z^{*}(w) \approx\left[1-A\left(w-w_{c}\right)^{1 /(\alpha-1)}\right] \mu_{3}^{-1}
$$

where $A$ is a constant. Then the average fraction of adsorbed monomers is $n \propto(\epsilon-$ $\left.\epsilon_{c}\right)^{1 /(\alpha-1)-1}$. A comparison with the well known scaling relationship $n \propto\left(\epsilon-\epsilon_{c}\right)^{1 / \phi-1}$ where $\phi$ is the so called adsorption (or, crossover) exponent [10] suggests that

$$
\phi=\alpha-1
$$

This is a result of principal importance. It shows that the crossover exponent $\phi$, describing polymer adsorption at criticality, is determined by the exponent $\alpha$ which governs polymer loop statistics! If loops are treated as isolated objects, then $\alpha=1-\gamma_{11} \approx 1.39$ so that $\phi=0.39$. In contrast, excluded volume interactions between a loop and the rest of the chain lead to an increase of $\alpha$ and $\phi$, as we show below.

Probability distributions of loops and tails - How does the length distribution of polymer loops and tails close to the CAP look like? From the expression for $U(z)$, given above, and eq.(3) we have $P_{\text {loop }} \approx\left(\mu_{3} z^{*}\right)^{l} / l^{1+\phi} \approx \exp \left[-c_{1}\left(\epsilon-\epsilon_{c}\right)^{1 / \phi}\right] / l^{1+\phi}$. This is valid only for $\epsilon>\epsilon_{c}$ since a solution for eq.(2) for subcritical values of the adhesive potential $\epsilon$ does not exist. Nontheless, even in the subcritical region, $\epsilon<\epsilon_{c}$, there are still monomers which 
occasionally touch the substrate, creating thus single loops at the expense of the tail length. The partition function of such a loop-tail configuration is $Z_{l-t}=\frac{\mu_{3}^{l}}{l^{1+\phi}} \frac{\mu_{3}^{N-l}}{(N-l)^{\beta}}$. On the other side, the partition function of a tail conformation with no loops whatsoever (i.e., of a nonadsorbed tethered chain) is $Z_{t}=\mu_{3}^{N} N^{\gamma_{1}-1}$. Thus the probability $P_{\text {loop }}^{<}(l)$ to find a loop of length $l$ next to a tail of length $N-l$ can be estimated as $P_{\text {loop }}^{<}(l)=\frac{Z_{l-t}}{Z_{t}} \propto \frac{N^{1-\gamma_{1}}}{l^{1+\phi}(N-l)^{\beta}}$, which is valid at $\epsilon<\epsilon_{c}$. In the vicinity of the CAP, $\epsilon \approx \epsilon_{c}$, the distribution will be given by an interpolation between the expressions above. Hence, the overall loop distribution becomes

$$
P_{\text {loop }}(l)= \begin{cases}\frac{1}{l^{1+\phi}} \exp \left[-c_{1}\left(\epsilon-\epsilon_{c}\right)^{1 / \phi} l\right], & \epsilon>\epsilon_{c} \\ \frac{A_{1}}{l^{1+\phi}}+\frac{A_{2} N^{1-\gamma}}{l^{1+\phi}(N-l)^{\beta}}, & \epsilon=\epsilon_{c} \\ \frac{N^{1-\gamma_{1}}}{l^{1+\phi}(N-l)^{\beta}} . & \epsilon<\epsilon_{c}\end{cases}
$$

The same reasonings for a tail leads to the distribution

$$
P_{\text {tail }}(l)= \begin{cases}\frac{1}{l^{\beta}} \exp \left[-c_{1}\left(\epsilon-\epsilon_{c}\right)^{1 / \phi} l\right], & \epsilon>\epsilon_{c} \\ \frac{B_{1}}{l^{\beta}}+\frac{B_{2} N^{1-\gamma_{1}}}{l^{\beta}(N-l)^{1+\phi}}, & \epsilon=\epsilon_{c} \\ \frac{N^{1-\gamma_{1}}}{l^{\beta}(N-l)^{1+\phi}} . & \epsilon<\epsilon_{c}\end{cases}
$$

In eqs.(5) - (6) $A_{1}, A_{2}, B_{1}, B_{2}$ are constants. Close to CAP these distributions are expected to attain a $\mathrm{U}$ - shaped form (with two maxima at $l=1$ and $l \approx N$ ), as predicted for a Gaussian chain by Gorbunov et al. [13]. For the average loop length $L$ the GCE-partition function for loops yields $L=z \partial U(z) /\left.\partial z\right|_{z=z^{*}}=\Phi\left(\alpha-1, \mu_{3} z^{*}\right) / \Phi\left(\alpha, \mu_{3} z^{*}\right)$. Close to the CAP, $L$ diverges as $L \propto 1 /\left(\epsilon-\epsilon_{c}\right)^{1 / \phi-1}$. The average tail length $S$ can be obtain as $S=$ $z \partial Q(z) /\left.\partial z\right|_{z=z^{*}}=\Phi\left(\beta-1, \mu_{3} z^{*}\right) /\left[1+\Phi\left(\beta, \mu_{3} z^{*}\right)\right]$. Again, using the properties of the polylog function, one can show that close to $\epsilon_{c}$ the average tail length diverges as $S \propto 1 /\left(\epsilon-\epsilon_{c}\right)^{1 / \phi}$. Note that this behavior corresponds to a length of adsorption blob $g \propto 1 /\left(\epsilon-\epsilon_{c}\right)^{1 / \phi}$.

Role of interacting loops and tails - Consider the number of configurations of a tethered chain in the vicinity of the CAP as an array of loops which end up with a tail. Using the approach of Kafri et al. [7] along with Duplantier's [9] graph theory of polymer networks, one may write the partition function $Z$ for a chain with $\mathcal{N}$ building blocks: $\mathcal{N}-1$ loops and a tail. Consider a loop of length $M$ while the length of the rest of the chain is $K$, that is, $M+K=N$. In the limit of $M \gg 1, K \gg 1$ (but with $M / K \ll 1$ ) one can show [14] that $Z \sim \mu_{3}^{M} M^{\gamma_{\mathcal{N}}^{s}-\gamma_{\mathcal{N}-1}^{s}} \mu_{3}^{K} K^{\gamma_{\mathcal{N}-1}^{s}-1}$ where the surface exponent $\gamma_{\mathcal{N}}^{s}=2-\mathcal{N}(\nu+1)+\sigma_{1}+\sigma_{1}^{s}$ 
and $\sigma_{1}, \sigma_{1}^{s}$ are critical bulk and surface exponents [9]. The last result indicates that the effective loop exponent $\alpha$ becomes

$$
\alpha=\gamma_{\mathcal{N}-1}^{s}-\gamma_{\mathcal{N}}^{s}=\nu+1
$$

Thus, $\phi=\alpha-1=\nu=0.588$, in agreement with earlier Monte Carlo findings [15]. One should emphasize, however, that the foregoing derivation is Mean-Field-like ( $Z$ appears as a product of loop- and rest-of-the-chain contributions) which overestimates the interactions and increases significantly the value of $\alpha$, serving as an upper bound. The value of $\alpha$, therefore, is found to satisfy the inequality $1-\gamma_{11} \leq \alpha \leq 1+\nu$, i.e., depending on loop interactions, $0.39 \leq \phi \leq 0.59$.

Adsorption under detaching force - Using the GCE approach now we treat the case of self-avoiding polymer chain adsorption in the presence of pulling force, thus extending the consideration of Gaussian chains by Gorbunov et al. [16]. Under external detaching force $f$, the tail GCE-partition function $Q(z)$ in eq. (1) has to be replaced by $\tilde{Q}(z)=1+\sum_{n=1}^{\infty}\left[\left(\mu_{3} z\right)^{n} / n^{\beta}\right] \int d^{3} r P_{n}(\mathbf{r}) \exp \left(f r_{\perp} / T\right)$ where $P_{n}(\mathbf{r})$ is the end-to-end distance distribution function for a self-avoiding chain [17]. After some straightforward calculations the tail GCE-partition function can be written as

$$
\tilde{Q}(z)=1+a_{1} \tilde{f}^{\theta} \Phi\left(\psi, z \mu_{3} \exp \left(a_{2} \tilde{f}^{1 / \nu}\right)\right)
$$

Here the dimensionless force $\tilde{f}=f a / k_{B} T$, the exponents $\psi=1-\nu$, and $\theta=(2+t-$ $3 \delta / 2) /(\delta-1)$ with $t=(\beta-3 / 2+3 \nu) /(1-\nu)$ and $\delta=1 /(1-\nu)$. The function $\tilde{Q}(z)$ has a branch point at $z^{\#}=\mu_{3}^{-1} \exp \left(-a_{2} \tilde{f}^{1 / \nu}\right)$, i.e., $\tilde{Q}(z) \sim 1 /\left(z^{\#}-z\right)^{1-\psi}$. One may, therefore, conclude that the total GCE-partition function $\Xi(z)$ has two singularities on the real axis: the pole $z^{*}$, and the branch point $z^{\#}$. It is known (see, e.g., Sec. 2.4.3. in [11]) that for $N>>1$ the main contributions to $\Xi_{N}$ come from the pole and the branch singular points, i.e.,

$$
\Xi_{N} \sim C_{1}\left(z^{*}\right)^{-N}+\frac{C_{2}}{\Gamma(1-\psi)} N^{-\psi}\left(z^{\#}\right)^{-N}
$$

Thus, for large $N$ only the smallest of these points matters. On the other hand, $z^{*}$ depends on the dimensionless adsorption energy $\epsilon$ only (or, on $w=\exp (\epsilon)$ ) whereas $z^{\#}$ is controlled by the external force $\tilde{f}$. Therefore, in terms of the two control parameters, $\epsilon$ and $\tilde{f}$, the equation $z^{*}(\epsilon)=z^{\#}(\tilde{f})$ determines the critical line of transition between the adsorbed phase 
and the force-induced desorbed phase. In the following this line will be called detachment line. Below it, $f<f_{D}$, or above, $f>f_{D}$, either $z^{*}$ or $z^{\#}$, respectively, contribute to $\Xi_{N}$. The controll parameters, $\epsilon_{D}$ and $\tilde{f}_{D}$, which satisfy this equation, denote detachment energy and detachment force, respectively. On the detachment line the system undergoes a firstorder phase transition. The detachment line itself terminates for $\tilde{f}_{D} \rightarrow 0$ in the CAP, $\epsilon_{c}$,
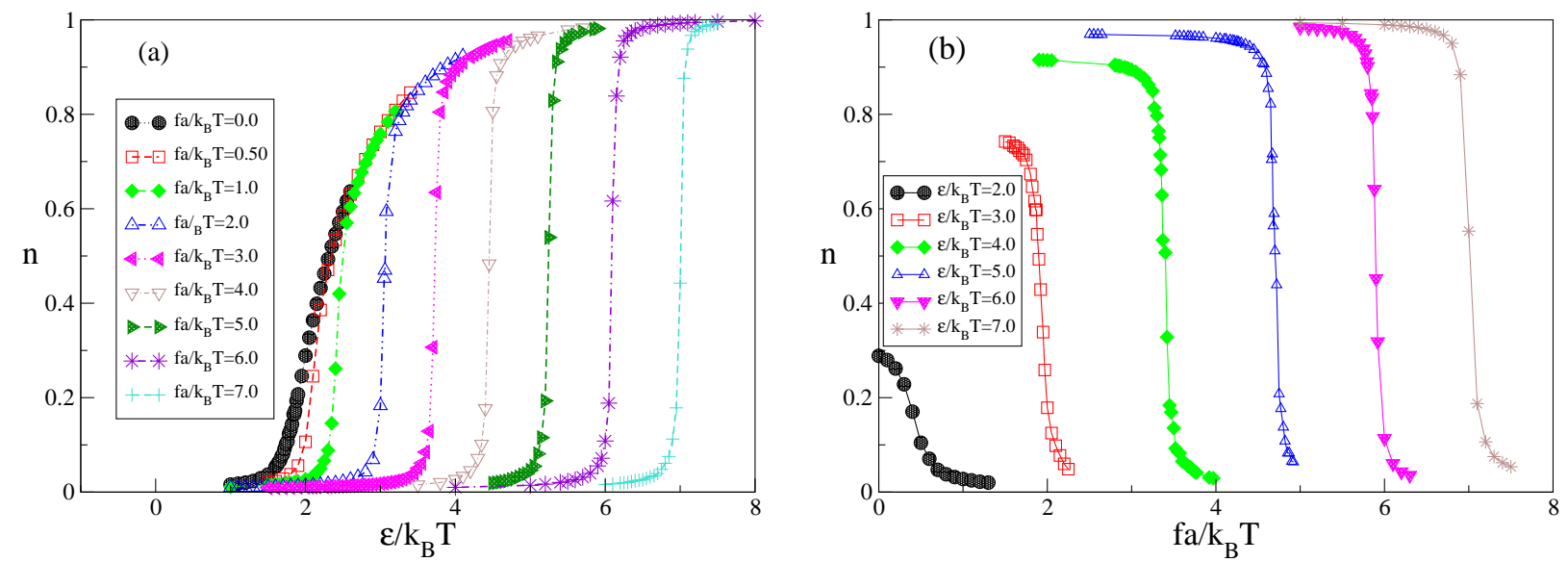

FIG. 1: (a) The 'order parameter', $n$, against the surface potential, $\epsilon$, for various pulling forces. The chain has length $N=128$. (b) Variation of $n$ with the pulling force, $f$, for several surface potentials.

force $\tilde{f}_{D}$ vanishes as $\tilde{f}_{D} \sim\left(\epsilon-\epsilon_{c}\right)^{\nu / \phi}$. This first order adsorption-desorption phase transition under pulling force has a clear dichotomic nature (i.e., it follows an "either - or" scenario): in the thermodynamic limit $N \rightarrow \infty$ there is no phase coexistence! The configurations are divided into adsorbed and detached (or stretched) dichotomic classes. Metastable states are completely absent. Moreover, the mean loop length $L$ remains finite upon detachment line crossing. The average tail length $S$, on the contrary, diverges close to the detachment line. Indeed, at $\tilde{f}<\tilde{f}_{D}$ the average tail length is given by $S=\tilde{f}^{\theta} \Phi\left(\psi-1, z^{*}(w) / z^{\#}(\tilde{f})\right) /[1+$ $\left.a_{1} \Phi\left(\psi, z^{*}(w) / z^{\#}(\tilde{f})\right)\right]$. At the detachment line, $z^{*}=z^{\#}$, it diverges as $S \propto \tilde{f}_{D} /\left(\tilde{f}_{D}-\tilde{f}\right)$.

Reentrant phase behavior - Recently, it has been realized [18] that the detachment line, when represented in terms of dimensional variables, force $f_{D}$ versus temperature $T$, goes (at a relatively low temperature) through a maximum, that is, the desorption transition shows reentrant behavior! Below we demonstrate that this result follows directly from our theory. It can be seen that the solution of eq.(2) at large values of $\epsilon$ (or, at low 


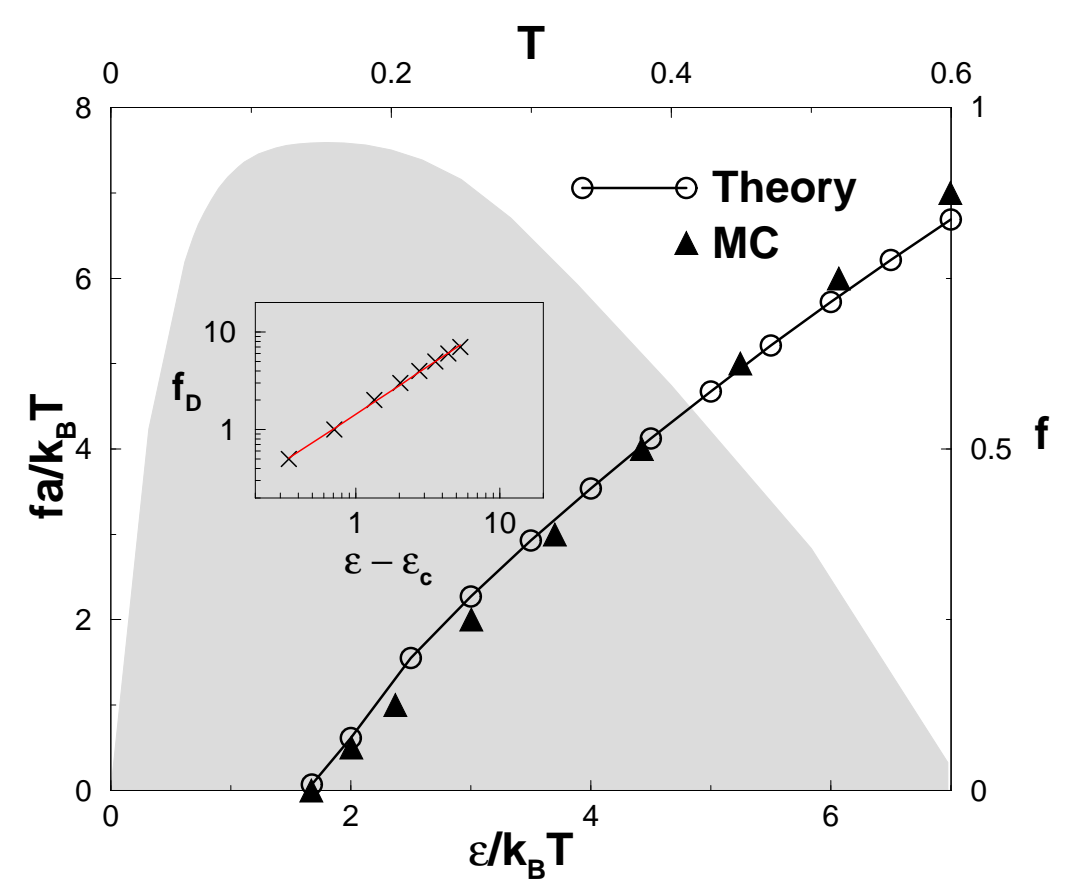

FIG. 2: Plot of the critical detachment force $f_{D}=f a / k_{B} T$ against the surface potential $\varepsilon / k_{B} T$. Full and empty symbols denote MC and theoretical results. A double logarithmic plot of $f_{D}$ against $\epsilon-\epsilon_{c}$ with $\epsilon_{c}=1.67$ is shown in the inset, yielding a slope of $0.97 \pm 0.02$, in agreement with the prediction $f_{D} \propto\left(\epsilon-\epsilon_{c}\right)^{\nu / \phi}$. Shaded is shown the same phase diagram, derived by numeric solution of $z^{*}=z^{\#}$, which in dimensional $f$ (right axis) against $T$ (top axis) units appears reentrant.

temperature) can be written as $z^{*} \approx \mathrm{e}^{-\epsilon} / \mu_{3}$ so that the detachment line, $z^{*}=z^{\#}$, in terms of dimensionless parameters is monotonous, $\tilde{f}_{D} \propto\left[\epsilon_{D}-\ln \left(\mu_{3} / \mu_{2}\right)\right]^{\nu}$. Note, however, that the same detachment line, if represented in terms of the dimensional control parameters, force $f_{D}$ versus temperature $T_{D}$ (with a fixed dimensional energy $\varepsilon_{0}$ ), shows a nonmonotonic behavior $f_{D}=T_{D}\left[\varepsilon_{0} / T_{D}-\ln \left(\mu_{3} / \mu_{2}\right)\right]^{\nu} / a$. This curve has a maximum at a temperature given by $T_{D}^{\max }=(1-\nu) \varepsilon_{0} / \ln \left(\mu_{3} / \mu_{2}\right)$.

Monte Carlo Simulation - We have investigated the force induced desorption of a polymer by means of extensive Monte Carlo simulations using a coarse grained off-lattice bead-spring model [19] of a polymer. Fig. 17a shows the variation of the order parameter $n$ (average fraction of adsorbed monomers) with changing adhesive potential $\epsilon$ at fixed pulling force whereas Fig 1 depicts $n$ vs. force $f a / T$ for various $\epsilon$. The abrupt change of the order parameter is in close agreement with our theoretical prediction. Using the threshold values of $f_{D}$ and $\epsilon_{D}$ for critical adsorption/desorption in the thermodynamic limit $N \rightarrow \infty$, one can 
construct the adsorption - desorption phase diagram for a polymer chain under pulling shown in Fig.2 which is among the central results of this work. The detachment lines, obtained from MC data and the numerical solution of $z^{*}=z^{\#}$ almost coincide, and the slope of $f_{D}$ vs $\left(\epsilon-\epsilon_{c}\right)$ is close to unity, according to the prediction $f_{D} \propto\left(\epsilon-\epsilon_{c}\right)^{\nu / \phi}$. Also indicated by the shaded area in Fig,2 is the reentrant image of the same phase diagram, obtained when the numerical solution of $z^{*}=z^{\#}$ is plotted in dimensional units $f$ versus $T$. In Fig. 3 a
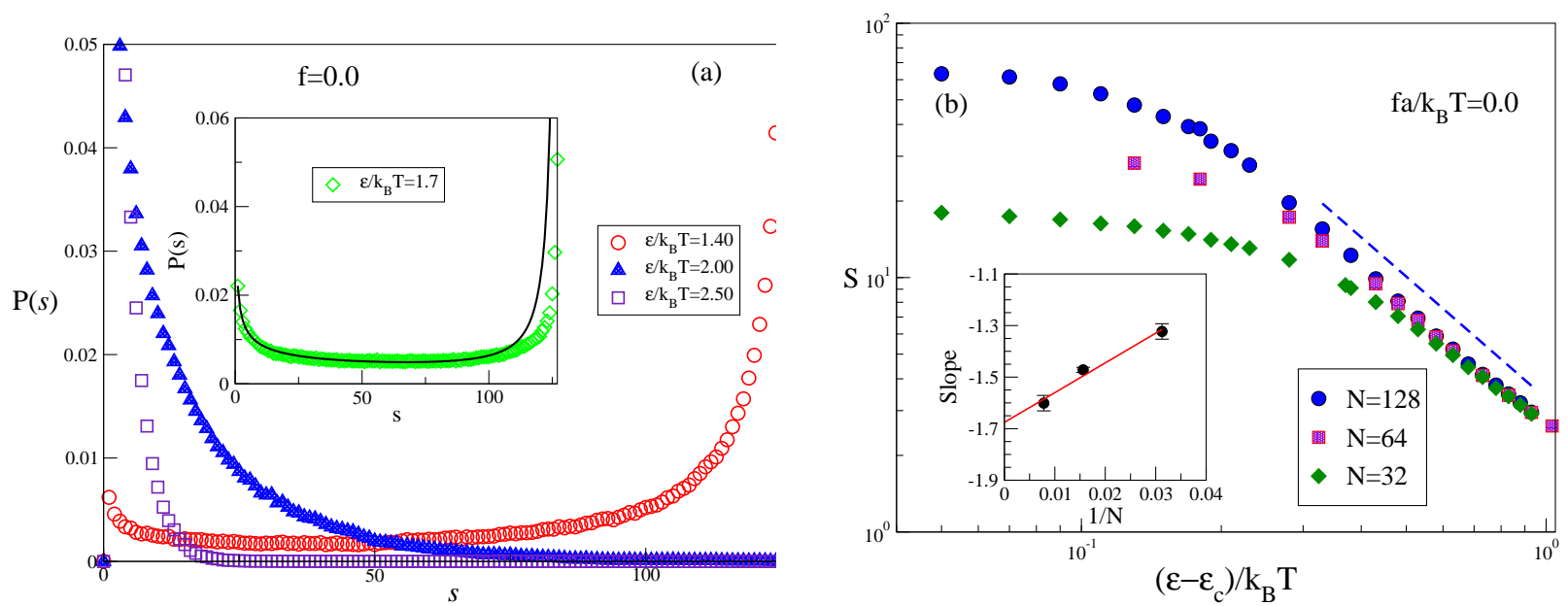

FIG. 3: (a) Tail length distribution $P(s)$ for different surface potentials close to $\epsilon_{c}$ in a polymer of length $N=128$ with no pulling force. In the inset $P(s)$ at $\epsilon=\epsilon_{c}$ (symbols) is compared to the prediction, Eq. (6) (full line). (b) Average tail length $S$ against $\left(\epsilon-\epsilon_{c}\right) / k_{B} T$ plotted for various chain lengths in log-log coordinates. The slopes of these curves are plotted against $1 / N$ in the inset and extrapolate to $1 / \phi$ in the thermodynamic limit $N \rightarrow \infty$.

we show the PDF of tail length at different strength of adsorption in the absence of pulling. This confirms the $\mathrm{U}$ - shape of $P(s)$ predicted by eq.(6). While for $s \rightarrow 1$ the agreement with eq. 6 is perfect, for $s \rightarrow N$ long tails are slightly overestimated by eq.(6). This small discrepancy reflects the dominance of our "single loop \& tail" approximation - multiple loops would effectively reduce the tail size. Fig. $3 \mathrm{~b}$ shows the divergency of $S$ close to the critical point $\epsilon_{c}$. For chain of finite length $N$, the tail length divergence at $\epsilon \rightarrow \epsilon_{c}$ is replaced by a rounding into a plateau since $S \rightarrow N$ but away from $\epsilon_{c}$ the measured slope extrapolates to the theoretical prediction $S \propto 1 /\left(\epsilon-\epsilon_{c}\right)^{1 / \phi}$. In the presence of pulling force one observes a remarkable feature of the order parameter probability distribution - an absence of two peaks 


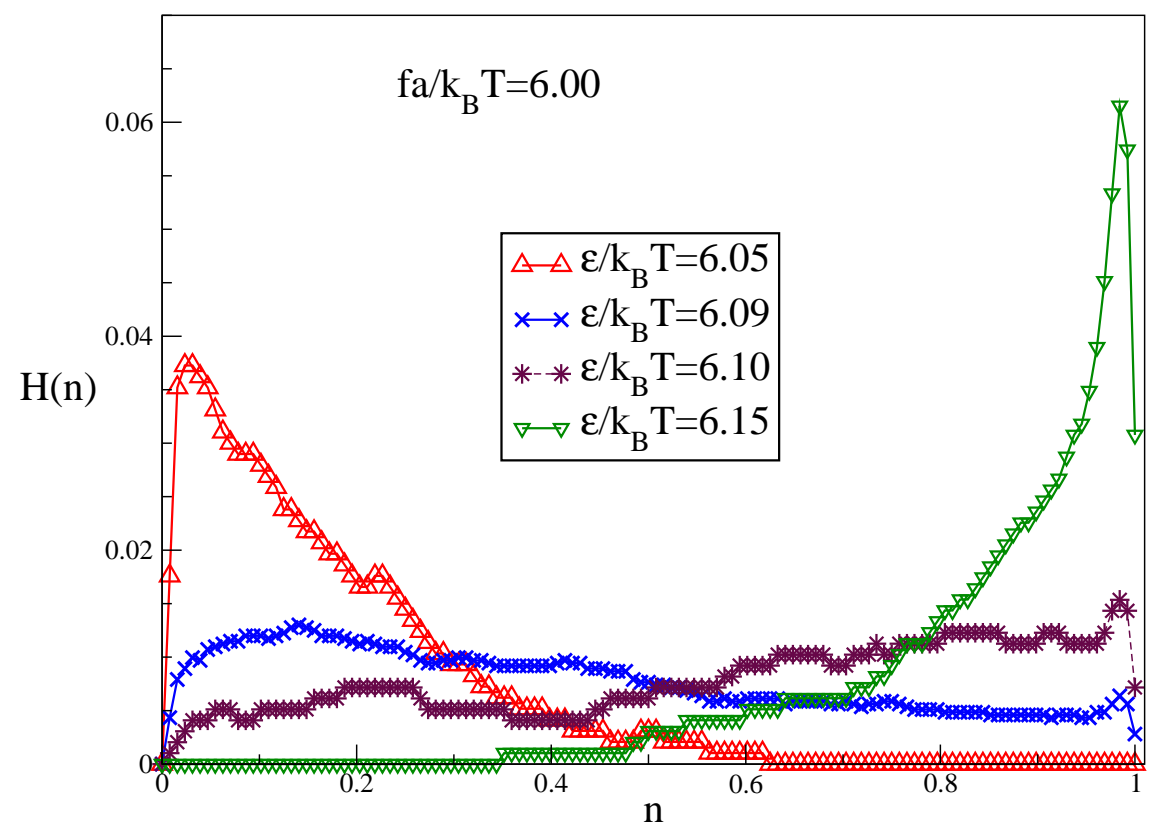

FIG. 4: Distribution of the order parameter $n$ for a pulling force $f a / k_{B} T=6.0$ an different strengths of adhesion $\epsilon / k_{B} T$. The chain length is $N=128$ and the threshold value of the surface potential for this force is $\epsilon_{D} \approx 6.095 \pm 0.03$. The values $\epsilon / k_{B} T=6.09$ and $\epsilon / k_{B} T=6.10$ are on both sides of the detachment line, cf. Fig. 2.

in the vicinity of the critical strength of adsorption, $\epsilon_{D} \approx 6.095 \pm 0.03$, which still keeps the polymer adsorbed at pulling force $f a / k_{B} T=6.0$ - Fig. 4. At $\epsilon_{D}$ the distribution $H(n)$ is flat, indicating huge fluctuations so that any value of $n$ is equally probable. Close to $\epsilon_{D}$, one observes a clear maximum in the distribution $H(n)$, indicating a desorbed chain with $n \approx 0.01$ for $\epsilon=6.05$, or a completely adsorbed chain with $n \approx 0.99$ for $\epsilon=6.15$. This lack of bimodality in the $H(n)$ manifests the dichotomic nature of the desorption transition which rules out phase coexistence.

In conclusion, we have demonstrated that a full description of the force induced polymer chain desorption transition can be derived by means of the GCE approach, yielding the average size and probability distribution functions of all basic structural units as well as their variation with changing force or strength of adhesion. The detachment transition is proved to be of first order albeit dichotomic in nature thus ruling out phase coexistence. The critical line of desorption, while monotonous when plotted in dimensionless units of detachment force against surface potential, becomes non-monotonous in units of force against temperature, 
thus outlining a reentrant phase diagram. In addition, we show that the crossover exponent, $\phi$, governing polymer behavior at criticality, depends essentially on interactions between different loops so that $0.39 \leq \phi \leq 0.59$. All these predictions appear in very good agreement with our Monte Carlo computer simulation results.

Acknowledgments We are indebted to A. Skvortsov, L. Klushin, J.-U. Sommer, and K. Binder for useful discussions during the preparation of this work. A. Milchev thanks the Max-Planck Institute for Polymer Research in Mainz, Germany, for hospitality during his visit in the institute. A. Milchev and V. Rostiashvili acknowledge support from the Deutsche Forschungsgemeinschaft (DFG), grant No. SFB 625/B4.

[1] T. Strick, et al. Phys. Today, 54, 46(2001).

[2] F. Celestini, et al. Phys. Rev. 70, 012801(2008).

[3] M. Rief, et al., Science, 275, 1295 (1997).

[4] S. B. Smith, et al., Science, 271, 795 (1996).

[5] B. J. Haupt, et al. Langmuir, 15, 3868 (1999).

[6] A. M. Skvortsov, et al., Polymer Sci. A (Moscow) (2009).

[7] Y. Kafri, et al. Eur. Phys. J. B 27, 135 (2002).

[8] D. Poland, H.A. Scheraga, J. Chem. Phys. 45, 1456 (1966).

[9] B. Duplantier, J. Stat. Phys. 54, 581 (1989).

[10] C. Vanderzande, Lattice Model of Polymers, Cambridge University Press, Cambridge, 1998.

[11] J.A. Rudnick, G.D. Gaspari, Elements of the random walk, Cambridge University Press, Cambridge, 2004.

[12] A. Erdélyi, Higher transcendental functions, v.1, N.Y., 1953.

[13] A. A. Gorbunov, et al. J. Chem. Phys. 114, 5366 (2001).

[14] S. Bhattacharya et al., submitted for publication.

[15] E. Eisenriegler, K. Kremer, K. Binder, J. Chem. Phys. 77, 6296 (1982).

[16] A.A. Gorbunov, A.M. Skvortsov, J. Chem. Phys. 98, 5961 (1993).

[17] J. des Cloizeaux, G. Jannink, Polymers in Solution, Clarendon Press, Oxford, 1990.

[18] P.K. Mishra, et al. Europhys. Lett. 69, 102 (2005).

[19] K. Binder and A. Milchev, J. Computer-Aided Material Design, 9, 33(2002). 